\title{
Probiotication of Bifidobacterium Spp. in dairy and non-dairy mango juice medium-A Green approach
}

\author{
Oorjitha Dogiparthi and Dorathy Pushparani
}

Received: 27 January 2021 / Accepted: 18 June 2021 / Published online: 31 October 2021

(C) Indian Dairy Association (India) 2021

\begin{abstract}
Mango (Mangifera indica L.) is a climacteric, tropical drupe of the Anacardiaceae family. This study aims at determining the ideal synbiotic combination using mango as a natural prebiotic source in two different mediums, dairy, and nondairy, for two different probiotics Bifidobacterium infantis and Bifidobacterium lactis. Skimmed milk and sugarcane were observed on analysis to promote the growth of these probiotics and were the chosen dairy and non-dairy substrates used in combination with mango. The ideal synbiotic combination of Mango with dairy /non-dairy medium was optimized on the basis of sensory evaluation supported by total viable counts. Fermentation for 4 hours of the ideal synbiotic combinations with $0.1 \%$ inoculum of probiotic cultures was done. The 03:07 ratio of mango: skimmed milk and mango: sugarcane with 4 hours of incubation gave desirable results with increasing microbial counts, drop in $\mathrm{pH}$ and nominal increase in acidity as fermentation time progressed. This ideal combination of Probiotics with Mango in both dairy and non-dairy medium could be a promising blend in the food and beverage industry for formulating naturally functional products.
\end{abstract}

Department of Food Chemistry \& Food Processing, Loyola College, Chennai-34, Tamil Nadu, India

Dorathy Pushparani $(\square)$

Department of Food Chemistry and Food Processing, Loyola College,Chennai-34, Tamil Nadu, India.

E-mail: dorothychris25@gmail.com
Keywords: Beverage, Green approach, Mango, Prebiotics, Probiotics, Synbiotics, Sugarcane juice, Skim milk

\section{Introduction}

Consumers show interest in ingesting diets to maintain the health and prevent the degenerative or chronic diseases such as diabetes, cancer, hypertension (Granato et al. 2010). The growing awareness of the relationship between diet and health has led to an increasing demand for food products that support health above and beyond providing basic nutrition i.e., functional foods. Functional foods comprise of conventional foods containing naturally occurring bioactive substances, foods enriched with bioactive substances and synthesized food ingredients introduced to traditional foods (Grajek et al. 2005). Prebiotics and probiotics are becoming leaders in the functional foods category as it is considered to exert positive effects on our body (Prado et al. 2008).

Probiotics have been a functional food of interest since the earliest of times. Probiotics are nonpathogenic microorganism which improves the intestinal microbial balance, provides health benefits and prevents some diseases (FAO, 2002). Prebiotics are non-digestible dietary components that pass through the digestive tract to the colon and are a potential substrate for fermentation by the microbiota. High potential is attributed to the simultaneous use of probiotics and prebiotics. The term "synbiotic" was first used in 1995, to describe a combination of synergistically acting probiotics and prebiotics (Bengmark, S., 2005).

Fruits \& vegetables such as banana, asparagus, beans, cereals, onion, garlic, Jerusalem artichoke, chicory, are natural prebiotic sources. Apart from being a prebiotic source, fruits and vegetables also have other functional properties which is an added benefit. Moreover, the fruit juices also have a good refreshing taste and are a consumption choice for people of all age groups since they are perceived as healthy foods. (Jankovic, Sybesmaet.al., 2010).

With the idea of utilizing locally available indigenous fruit as a natural prebiotic source, this study explores the use of mango in 
dairy and non-dairy medium as a substrate for growth of Bifidobacterium species. Mango could be a better, greener and safer alternative as a prebiotic for use in the ideal synbiotic combination.

This study aims at determining the ideal synbiotic combination using mango as a natural prebiotic source in two different mediums, dairy, and non-dairy, for the probiotics Bifidobacterium infantis and Bifidobacterium lactis

\section{Materials and Methods}

\section{Materials}

Fresh mangoes were procured from the local market (Banganapalli variant). Fresh sugarcane juice was purchased from the local vendor and stored under refrigeration condition prior to use. Branded skim milk in tetra pack was procured from the supermarket and used for the study.

\section{Probiotic strain}

Proven probiotic strains of Bifidobacterium infantis (UBBI-01) \& Bifidobacterium lactis (UBBLa-70) was obtained in the lyophilized forms from Unique biotech, Hyderabad for the study.

\section{Probiotic Culture}

The cultures were prepared by incubating the probiotic strains in Bifidobacterium broth (Himedia No. M1395) overnight at $37^{\circ} \mathrm{C}$ under anaerobic conditions to obtain the working culture. This was maintained at $5^{\circ} \mathrm{C}$ and sub cultured in Bifidobacterium broth.

\section{Total Plate Count-TPC}

Dilutions were placed on selective Bifidobacterium agar (Himedia No. M1396) and grown under anaerobic conditions in Anaerobic jars (GasPak System) for 48 hours at $37^{\circ} \mathrm{C}$ (Vinderola \& Reinheimer, 1999).

\section{Turbidimetric method}

Turbidimetric method is an indirect, rapid method used for following growth of bacterial cultures. A spectrophotometer can be used for measurement of cell mass and in this indirect determination method the absorbances were estimated before and after incubation (Pelczar M. J.et al. ,2003). The optical density of the samples was checked at the wavelength of $600 \mathrm{~nm}$

\section{Gram staining}

Representative colonies were taken and subjected to Grams staining techniques (RajanS. \& Christy R., 2010) and observations were recorded.

\section{Assessing the chosen dairy and non-dairy substrates for promoting Probiotic growth}

Skimmed milk and sugarcane juice were checked for their suitability as a potential dairy and non-dairy substrate for $B$. lactis and $B$. infantis, by inoculating the probiotic cultures $(100$ $\mu \mathrm{l}$ in $10 \mathrm{ml}$ of substrate), incubating for 24 hours and determining the bacterial viable counts and change in absorbance.

\section{Assessing prebiotic efficacy of mango}

The prebiotic potential of mango was observed by inoculating probiotic cultures ( $100 \mu \mathrm{l}$ in $10 \mathrm{ml}$ of substrate), incubating for 24 hours and determining the bacterial viable count and change in absorbance.

\section{Sample Preparation}

The selected mango fruits (Banganapalli variant) were washed and cleaned with potable water. The inedible parts like seed, rind and peel were removed. The fruit pieces were ground in a blender and the pulp of the fruits were used in the study. The pulp was stored frozen for further use. Mango pulp, skimmed milk, sugarcane juice were individually heated at $80^{\circ} \mathrm{C}$ for 20 minutes and were used to prepare different combination.

\section{Optimization of the ideal blend ratio}

The dairy blend was prepared with skimmed milk and mango pulp, while the non-dairy blend was prepared with sugarcane juice and mango pulp in different ratios as given in Table 1

\section{Sensory evaluation}

The different ratios of fruit and the dairy medium/fruit and nondairy medium were subjected to sensory evaluation using a five points hedonic scale. The sensory attributes were evaluated by

Table 1: Blend ratios

\begin{tabular}{lllll}
\hline \multirow{3}{*}{ Blend ratio } & \multicolumn{4}{l}{ Blend ratio of mango based dairy medium } \\
\cline { 2 - 4 } Mango: Skimmed milk & D1 & D2 & D3 & D4 \\
& Blend ratio of mango based non-dairy medium & $04: 06$ \\
Blend ratio & ND1 & ND2 & ND3 & ND4 \\
Mango: Sugarcane juice & $01: 09$ & $02: 08$ & $03: 07$ & $04: 06$ \\
\hline
\end{tabular}


30 panelists. The best combination acceptable to the consumers was determined and the ideal ratio of mango: dairy, mango: nondairy was chosen for further study.

\section{Assessing the chosen substrates for promoting probiotic growth}

The viable count was determined by plate count technique. 100 $\mu l$ of the $B$. infantis was inoculated into all the combinations. The blends were enumerated on selective Bifidobacterium agar \&grown under anaerobic conditions in for 48 hours at $37^{\circ} \mathrm{C}$ (Vinderola \& Reinheimer, 1999). Turbidimetric technique was also used as the indirect method of measuring the growth (Pelczar et al. 2003). The viable count of B. lactis was also similarly determined by total plate count and turbidimetric technique.

\section{Analysis of the ideal synbiotic combination}

\section{Assessing the Growth pattern}

The ideal combination was freshly prepared and $0.1 \%$ of each probiotic culture was inoculated for the study. Viable counts and turbidity were determined at 0 hours and 4 hours.

\section{Measurement of specific chemical changes produced}

$\mathrm{pH}$ and acidity were determined by $\mathrm{pH}$ meter, titration (AOAC, 2016) respectively. Both $\mathrm{pH}$ and $\mathrm{TA}$ values of the formulated beverages was measured at 0,2 and 4 hours during the incubation period.

\section{PCR}

PCR is used a novel approach to confirm the probiotic growth in mango-based combinations.

Genomic DNA from the culture\& probiotic product was extracted using a DNeasy Tissue Kit (Qiagen, Hilden, Germany) according to the manufacturer's protocol Genomic DNA prepared was used as a template for PCR amplification. The amplification was done as mentioned by Yeon S, $2007 \&$ the amplified products were analyzed by electrophoresis on a $2 \%$ agarose gel containing $1 \mu 1$ of ethidium bromide, and the DNA bands were visualized under UV illumination and photographed

\section{Results and Discussion}

\section{Turbidimetric method}

The optical density of $B$. infantis and $B$. lactis before incubation was found to be $0.377 \pm 0.039,0.53 \pm 0.022$ and after incubation was found to be $0.721 \pm 0.108,0.928 \pm 0.017$ respectively (Figures 1 $\& 2)$.

TPC
Using the plate count method, B. infantis and B. lactis was found to have $9.9242 \pm 0.029,9.0492 \pm 0.062 \log \mathrm{cfu} / \mathrm{ml}$ in the working culture used in the study.

\section{Gram staining}

Representative colonies were taken and subjected to Grams staining techniques and it was observed that both $B$. infantis and $B$. lactis were stained purple, indicating that both are gram positive and rod shaped.

\section{Assessing the chosen substrates for promoting probiotic growth}

The ability of the selected dairy and non-dairy substrates (milk and sugarcane juice) to promote the growth of probiotics $B$. infantis and $B$. lactis individually was evaluated. It was observed that the viable count of $B$. infantis and $B$. lactis in the substrate milk, after 24 hours, was found to have increased from initial values of $9.9242 \pm 0.029,9.0492 \pm 0.062 \mathrm{log} \mathrm{cfu} / \mathrm{ml}$ to $11.1289 \pm 0.019$ and $10.3388 \pm 0.019 \log \mathrm{cfu} / \mathrm{ml}$ respectively. This was also supported by the increase in OD values determined by turbidimetric determination. The optical density of $B$. infantis and $B$. lactis in the substrate milk, before incubation was found to be $0.514 \pm 0.016,0.512 \pm 0.04$ and after incubation was $1.212 \pm 0.012$, $1.123 \pm 0.01$ respectively. Milk, the dairy medium used in Mangodairy combination is a proven substrate which promotes probiotic growth. Dairy-based fermented products and yogurts have been utilized as potential probiotic products since the ancient times (Bansal S et al. 2016).

The viable count of $B$. infantis and $B$. lactis in the substrate sugarcane was found to increase to $11.5021 \pm 0.080 \mathrm{log} \mathrm{cfu} / \mathrm{ml}$ and $10.7695 \pm 0.008 \log \mathrm{cfu} / \mathrm{ml}$ respectively. Similarly there was also an optical density of $B$. infantis and B. lactis in the substrate sugarcane, from $0.521 \pm 0.005$ to $1.618 \pm 0.006$ and $0.502 \pm 0.008$ to $1.311 \pm 0.005$ respectively.

An increase in the viable count of Bifidobacterium strains in native soymilk medium was observed by Havas et al. (2015). The fermentation processes cultured with initial cell concentrations in $10^{5}-10^{7} \mathrm{cfu} / \mathrm{ml}$ resulted in $10^{8} \mathrm{cfu} / \mathrm{ml}$ after $8-12 \mathrm{~h}$ of incubation in soymilk, and were kept viable up to the end of fermentation (48 h). Volumetric productivities of B. bifidum (B-3.2), B. bifidum (B7.1) \& B. breve (B-9.14) were $1.6 \times 10^{10} \mathrm{cfu} / \mathrm{L} . \mathrm{h}, 4.5 \times 10^{10} \mathrm{cfu} / \mathrm{L} . \mathrm{h}$ and $7.6 \times 10^{9} \mathrm{cfu} / \mathrm{L} . \mathrm{h}$, respectively. The values of $B$. lactis $(\mathrm{Bb}-12)$ $\& B$. longum $(\mathrm{Bb}-46)$ probiotic strains were $2.7 \times 10^{9} \mathrm{cfu} / \mathrm{L} . \mathrm{h}$ and $1.0 \times 10^{10} \mathrm{cfu} /$ L.h.

\section{Assessing prebiotic efficacy of mango}

The exact component responsible for mango's prebiotic capacity is unknown. Its prebiotic potential could due to the presence gallic acid, dietary fiber, resistant starch \& also pectin content (Yadav et al. 2010; Ramulu et al. 2003; Chatterjee et al. 2016; Holscher, 2017; Slavin, 2013). The Prebiotic potential of mango 


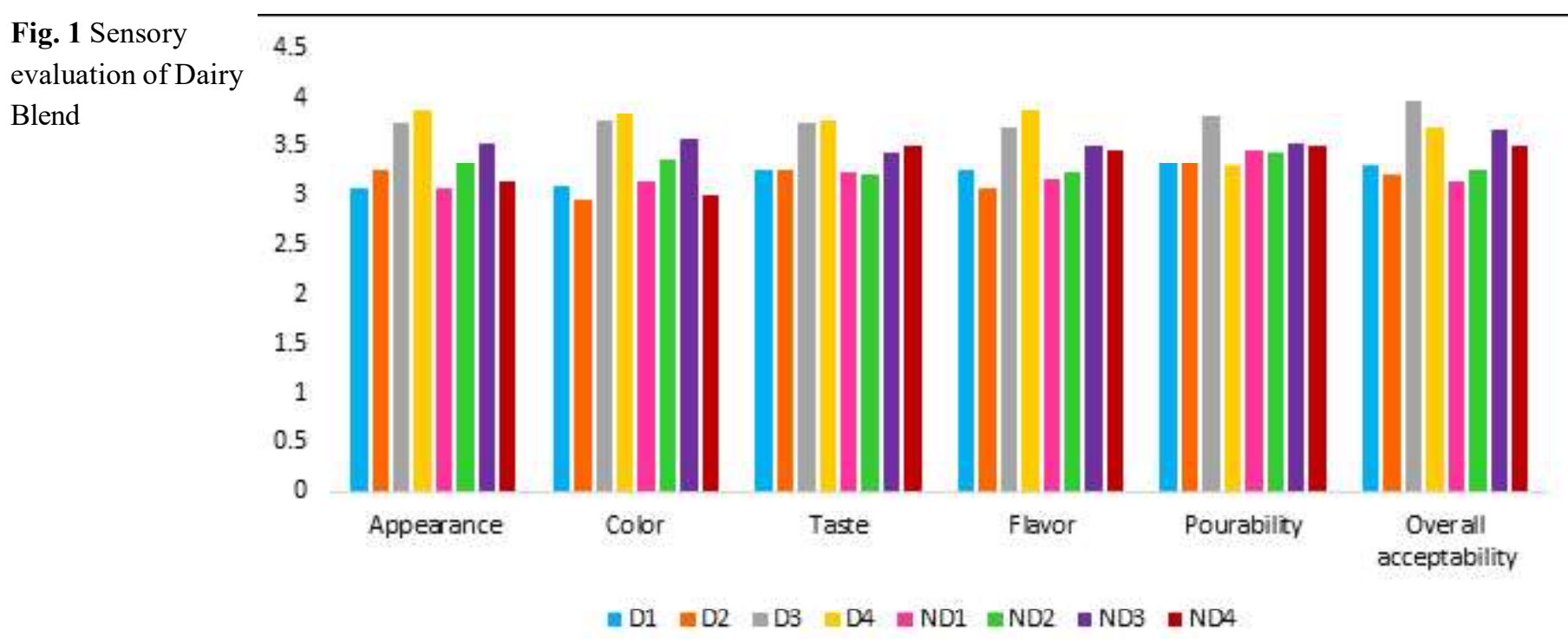

was observed by bacterial viable count and turbidimetric method. The viable count of $B$. infantis and $B$. lactis was found have increased to $11.2508 \pm 0.024$ and $10.3660 \pm 0.012 \mathrm{log} \mathrm{cfu} / \mathrm{ml}$ on incubation. This was also supported by the increase in OD values which was found to increase from $0.934 \pm 0.002$ to $1.458 \pm 0.006$ for B. infantis and from $1.222 \pm 0.01$ to $1.423 \pm 0.01$ for B. lactis.

\section{Optimization of the ideal blend ratio used in the study}

\section{Sensory evaluation}

Fruit juices have been suggested as an ideal medium for the functional health ingredients because they inherently contain beneficial nutrients, they have taste profiles that are pleasing to all the age groups (Vasudha and Mishra, 2013). It could be clearly observed that the panelists showed more preference as the proportion of mango juice increased ( figure 1).

Statistical analysis of the results revealed that significant difference existed between the overall acceptability score $(p<0.05)$ of samples between the different blend ratios in both dairy and non-dairy mediums.

\section{Assessing the ability of the probiotic culture to utilize the blends as a prebiotic substrate}

\section{Turbidimetric method}

The change in optical density before and after incubation for 24 hours, in the different mango-skim milk and mango-sugarcane juice blends inoculated with $B$. infantis was observed to increase and the highest of variations was found in D3I, ND3I.

Similarly the change in optical density in different mango-skim milk and mango-sugarcane juice blends inoculated with $B$. lactis was observed and the highest variations was seen in ND3I, ND3L (Table 2).

\section{TPC method}

After 24 hours increase in total viable count of B. infantis inoculated in the different mango-skim milk and mango-sugarcane juice blends was observed. It can be observed that D3I and ND3I show higher log cfu values (11.9173 \pm 0.003$),(12.0816 \pm 0.004)$. Statistical analysis showed that the viable count of dairy and non-dairy combination inoculated with $B$. infantis was found to have no significant difference, hence comparable.

Similarly, after 24 hours an increase in total viable count of $B$. lactis inoculated in the different mango-skim milk and mango sugarcane juice blends was observed. D3L and ND3L showed higher log cfu values (10.6117 \pm 0.003$),(11.0792 \pm 0.045)$ when compared to other samples (see Table 2). Statistical analysis showed that the viable count of dairy and non-dairy combination inoculated with $B$. lactis is found to have no significant difference, hence comparable.

The results of this study were similar with the results of study by Bujna et al. (2018) which showed that all investigated Bifidobacterium and Lactobacillus strains were able to grow well in the apricot juice without supplement of any nutrients meaning this matrix in itself was suitable medium for propagation of probiotic bacteria. In all cases the cell numbers at $24 \mathrm{~h}$ of fermentation were higher than $10^{8} \mathrm{cfu} / \mathrm{mL}$, and cell yields varied from $1.15 \times 10^{10} \mathrm{cfu} / \mathrm{L}$ h to $1.78 \times 10^{10} \mathrm{cfu} / \mathrm{L} \mathrm{h}$.

\section{Analysis of the ideal synbiotic combination}

The ideal synbiotic combination of 3:7 mango with dairy /nondairy medium was optimized on the basis of the sensory score, supported by highest total viable counts in this ratio. 
Fig. $2 \mathrm{pH}$

\& TA of

D3I,ND3I,

D3L and

ND3L
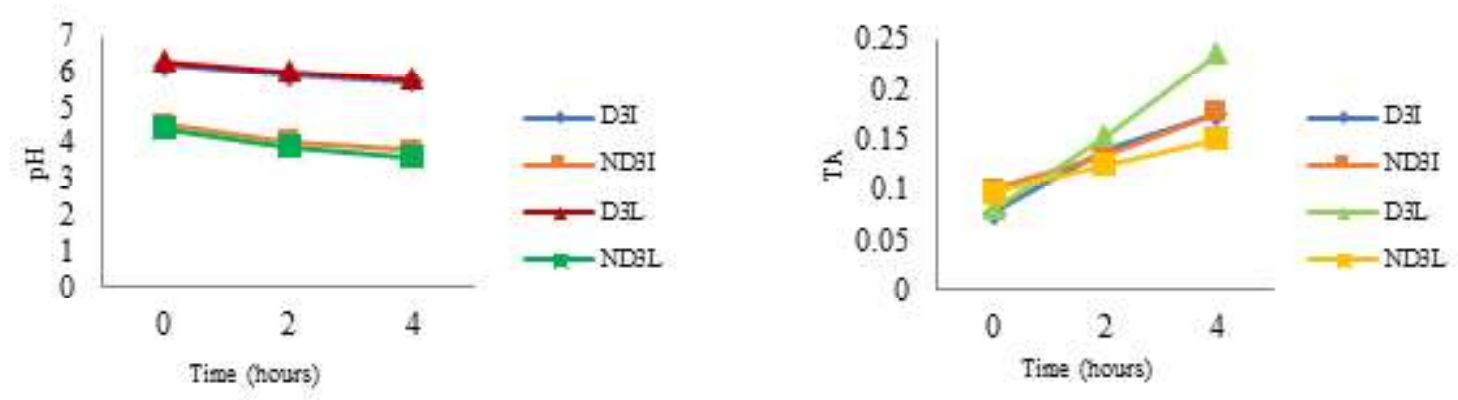

D3I- 03:07 mango skim milk blend inoculated with B.infantis; ND3I-03:07 mango sugarcane blend inoculated with B.infantis; D3L- 03:07 mango skim milk blend inoculated with B.lactis; ND3L-03:07 mango sugarcane blend inoculated with B.lactis

Table 2 Total plate count and variation in OD values of the dairy and non-dairy blends

\begin{tabular}{lll}
\hline & \multicolumn{2}{l}{ TPC } \\
\cline { 2 - 3 } & \multicolumn{1}{l}{ Dairy and non-dairy mango blends inoculated with B infantis } \\
\hline D1I & $10.6018 \pm 0.072$ & $0.167 \pm 0.002$ \\
D2I & $10.6086 \pm 0.030$ & $0.234 \pm 0.003$ \\
D3I & $11.9173 \pm 0.003$ & $0.808 \pm 0.007$ \\
D4I & $11.3848 \pm 0.018$ & $0.661 \pm 0.004$ \\
ND1I & $10.5433 \pm 0.032$ & $0.287 \pm 0.002$ \\
ND2I & $10.5289 \pm 0.057$ & $0.577 \pm 0.001$ \\
ND3I & $12.0816 \pm 0.004$ & $0.978 \pm 0.005$ \\
ND4I & $10.5595 \pm 0.035$ & $0.554 \pm 0.021$ \\
& Dairy and non-dairy mango blends inoculated with B.lactis & \\
D1L & $9.2709 \pm 0.014$ & $0.316 \pm 0.013$ \\
D2L & $9.3603 \pm 0.031$ & $0.451 \pm 0.006$ \\
D3L & $10.6117 \pm 0.003$ & $1.136 \pm 0.004$ \\
D4L & $10.4493 \pm 0.033$ & $0.516 \pm 0.02$ \\
ND1L & $9.4771 \pm 0.002$ & $0.42 \pm 0.011$ \\
ND2L & $9.4722 \pm 0.008$ & $0.21 \pm 0.003$ \\
ND3L & $11.0792 \pm 0.0457$ & $0.489 \pm 0.005$ \\
ND4L & $9.4771 \pm 0.001$ & $0.374 \pm 0.007$ \\
\hline
\end{tabular}

The values are the means of triplicate determination. \pm indicates the standard deviation of the mean

Table 3 OD values at $0^{\text {th }}$ hour and after 4 hours

\begin{tabular}{llc}
\hline & 0 Hour & 4 Hour \\
\hline D3I & $1.486 \pm 0.002$ & $1.726 \pm 0.004$ \\
ND3I & $1.444 \pm 0.003$ & $1.716 \pm 0.002$ \\
D3L & $1.536 \pm 0.002$ & $1.8775 \pm 0.001$ \\
ND3L & $1.417 \pm 0.001$ & $1.785 \pm 0.005$ \\
\hline
\end{tabular}

The values are the means of triplicate determination. \pm indicates the standard deviation of the mean

\section{Assessing the growth pattern}

The growth pattern of the culture in the combinations during 4 hours of incubation was assessed by determining colony forming unit and optical density at various time intervals. The colony count of D3I increased from $10.1785 \pm 0.024$ to $10.4413 \pm 0.010 \&$ for ND3I the colony count increased from $10.150 \pm 0.007$ to
$11.4771 \pm 0.001$. Similarly, the colony count of D3L increased from $9.7898 \pm 0.017$ to $10.2936 \pm 0.013 \&$ for ND3L the colony count increased from $9.7505 \pm 0.019$ to $10.3658 \pm 0.020$. The change in optical density of D3I, ND3I, D3L, ND3L from 0 hour to after 4 hours is shown in table 3.

In the study by Nguyen BT et al. (2019) pineapple juices were inoculated with different probiotic bacteria (Lactobacillus acidophilus, Lactobacillus plantarum, and Bifidobacterium lactis) at an initial cell density of about $10^{6}-10^{7} \mathrm{cfu} / \mathrm{ml}$. All investigated Lactobacillus and Bifidobacterium strains were able to grow well in the pineapple juice. In the case of bifidobacteria it reached a level of $10^{9} \mathrm{cfu} / \mathrm{ml}$ after 24 of fermentation.

\section{Physiochemical analysis: pH \& titrable acidity}


Fig. 3 PCR

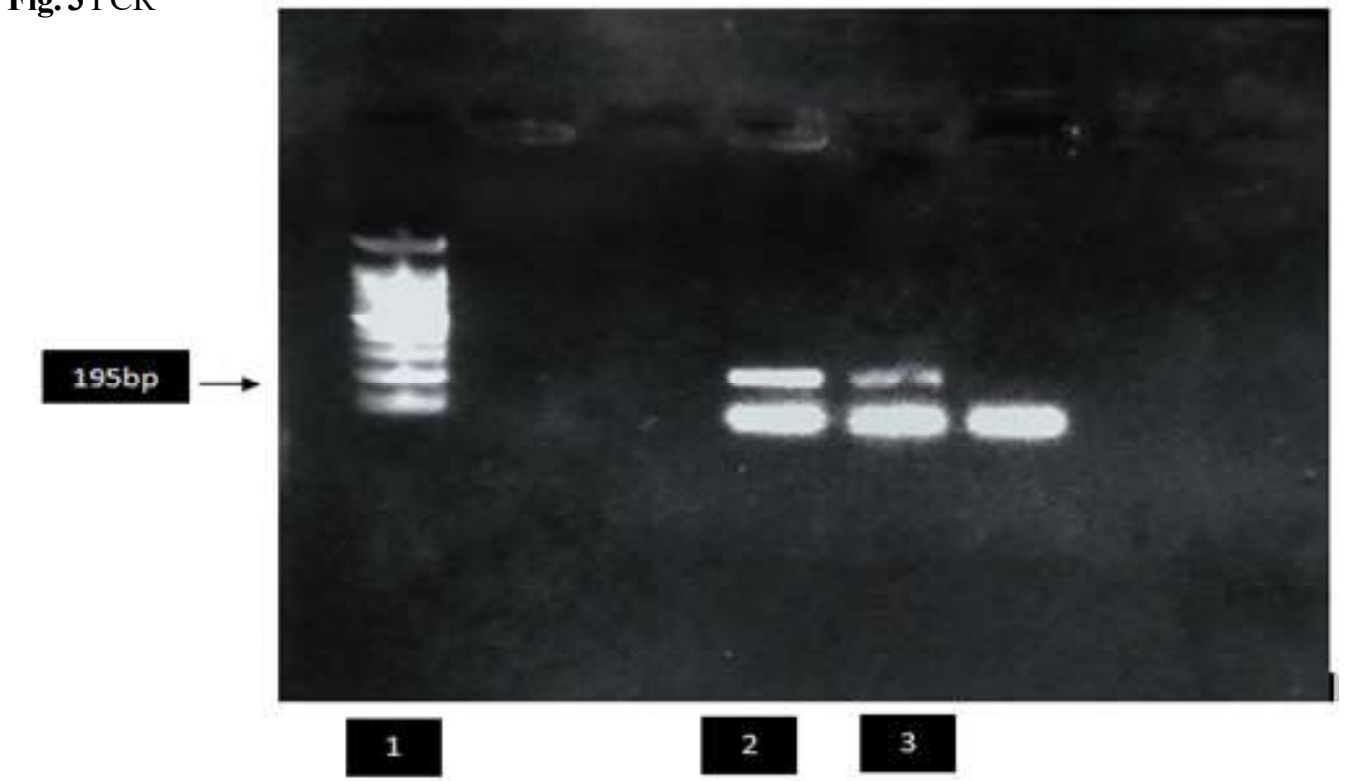

(1) 100bp ladder (2) Bifidobacterium lactis culture (3) Mango inoculated with $B$. lactis

Both $\mathrm{pH}$ and titrable acidity values of the formulated combinations were measured at 0,2 and 4 hours during the incubation period. The $\mathrm{pH}$ of all the four combinations was found to decrease gradually over the hours (shown in figure 2 ). The percentage TA of mango in dairy and non-dairy medium (B. infantis) observed at $0,2,4$ hours recorded an increase and for mango in dairy and non-dairy medium (B. lactis) the percentage TA was found to increase as incubation time increases (figure 2). Andrade et al (2020) has also reported a similar decrease in $\mathrm{pH}$ from the initial $5.28 \& 6.47$ of jerivá and macaúba palm fruit pulps to 4.76 and 5.20 respectively during the fermentation with $B$. lactis

The results obtained of increased $\log \mathrm{cfu} / \mathrm{ml}$ and acidity combined with drop in $\mathrm{pH}$ are indicative that 3:7 ratio of mango:skimmed milk and mango: sugarcane were utilized as a prebiotic source for growth by $B$. lactis and B. infantis.

\section{PCR}

The presence of probiotic culture B. lactis was confirmed using specific primers, by conventional PCR. The sequence of the $B$. lactis primer used is forward primer: CCCTTTCCACGGGTC-CC and reverse primer: AAGGGAAA-CCGTGTCTCCAC The PCR product was represented by a single band in the corresponding region of the DNA marker ladder (figure 3).

\section{Freeze dried powder of the ideal symbiotic combinations}

Freeze-drying is a technique adopted for successful long-term preservation of material. The 3:7 blend of the ideal synbiotic combinations were freeze dried and preserved for further use.

\section{Conclusion}

Mango as a prebiotic source in dairy and nondairy medium showed promising and comparable results for both the bifidobacterium species. The blend ratio of 3:7 for the both the mediums was observed to be the ideal synbiotic combination which was optimized on the basis of the sensory score, supported by highest total viable counts in this ratio. It was observed that the ideal synbiotic combination of mango: dairy and mango: nondairy medium containing $0.1 \%$ of $B$. infantis or B. lactis with a fermentation time of 4 hours provided desirable results for viable growth of probiotic culture and activity in terms of $\mathrm{pH}$ and acidity and hence can be used in probiotic product formulations using a green approach.

\section{Acknowledgement}

We acknowledge the Department of Biotechnology, TANUVAS for the support extended to carry out the research \& also Unique Biotech for providing us the culture.

\section{References}

Andrade AC, Marinho JF, Souza AC, Tavares TD, Dias DR, Schwan RF, Bastos SC (2020) Prebiotic potential of pulp and kernel cake from Jerivá (Syagrus romanzoffiana) and Macaúba palm fruits (Acrocomia aculeata). Food Res Int 136:109595

AOAC Official method 942.15. (2000) Acidity (Titrable) of fruit products. Official Methods of Analysis, 17th ed, A, Washington DC

AOAC Official method 981.12 (2000) $\mathrm{pH}$ of acidified foods. Official Methods of Analysis, 17th ed, A, Washington DC

Bajagai Y, Klieve A, Dart P, Bryden W (2016) Probiotics in animal nutrition - Production, impact and regulation, FAO Animal Prod Health Paper No. 179 
Bansal S, Mangal M, Sharma SK, Gupta RK (2016) Non-dairy Based Probiotics: A Healthy Treat for Intestine. Crit Rev Food Sci Nutr 56:1856-67

Bengmark S (2005) Bioecological control of the gastrointestinal tract: The role of flora and supplemented probiotics and synbiotics. Gastroenterol Clin North Am 34: 413-436

Bujna E, Farkas NA, Tran MA, Dam SM, Nguyen DQ (2018) Lactic acid fermentation of apricot juice by mono and mixed cultures of probiotic Lactobacillus and Bifidobacterium strains. Food Sci Biotechnol 27:547- 54

Chatterjee M, Manuel GAS, Hassan SS (2016) Effect of Fruit Pectin on Growth of Lactic Acid Bacteria. J Probiotic Health 4: 147

Grajek W, Olejnik A, Sip A (2005) Probiotics, prebiotics and antioxidants as functional foods. Acta Biochim Pol 52:665-71

Granato D, Branco GF, Nazzaro F, Cruz AG, Faria JA (2010) Functional Foods and Non-dairy Probiotic Food Development: Trends, Concepts, and Products. Compr Rev Food Sci F 9: 292-302

Havas P, Kun S, Perger-Mészáros I, Rezessy-Szabó, JM, Nguyen DQ (2015) Performances of new isolates of Bifidobacterium on fermentation of soymilk. Acta Microbiol Immunol Hung 62:463-476

Holscher HD (2017) Dietary fiber and prebiotics and the gastrointestinal microbiota. Gut Microbes 8:172-184

Jankovic I, Sybesma W, Phothirath P, Ananta E, Mercenier A (2010) Application of probiotics in food products - challenges and new approaches. Curr Opin Biotechnol 21: 175-181

Khatoon N, Gupta RK (2015) Probiotics beverages of sweet lime and sugarcane juices and its physiochemical, microbiological and shelflife studies. J Pharmacogn Phytochem 4: 25-34

Pelczar MJ, Chan EC, Krieg NR (2003) Microbiology (5 $5^{\text {th }}$ ed.) Tata McGraw-Hill Education Pvt. Ltd New York

Nguyen BT, Bujna E, Fekete N, Tran A, Rezessy-Szabo JM, Prasad R, Nguyen QD (2019) Probiotic Beverage from Pineapple Juice fermented with Lactobacillus and Bifidobacterium strains. Front Nutr $6: 54$
Prado FC, Prada JL, Pandey A, Soccol CR (2008) Trends in Non-Dairy Probiotic beverages. Food Res Int 41:111-123

Rajan S, Christy R (2010) Experimental procedures in life sciences. Anjanaa Book House

Ramulu P, Rao U (2003) Total, insoluble and soluble dietary fiber contents of Indian fruits. J Food Comp Anal16:677-685

Reddy LV, Min J, Wee Y (2015) Production of Probiotic Mango Juice by Fermentation of Lactic Acid Bacteria. Microbiol Biotechnol Lett 43: $120-125$

Slavin J (2013) Fiber and Prebiotics: Mechanisms and Health Benefits Nutrients. 1417-1435

Vasudha, S, H N Mishra (2013) Non-dairy Probiotic beverage. Int Food Res J 20:7

Vinderola CG, Reinheimer JA (1999) Culture media for the enumeration of Bifidobacterium bifidum and Lactobacillus acidophilus in the presence of yoghurt bacteria. Int Dairy J 9: 497-505

Yadav S, Gite S, Nilegaonkar S, Agte V (2010). Effect of supplementation of micronutrients and phytochemicals to fructooligosaccharides on growth response of probiotics and E. coli. J Sci Ind Res 37:58-64

Yeon S, SUL, Kim H, Kim T, Kim HY (2007) Rapid Identification of Lactobacillus and Bifidobacterium in Probiotic Products Using Multiplex PCR. J Microbiol 17: 490-495 